\title{
A STATISTICAL STUDY OF SHELL-MODEL EIGENVECTORS
}

\author{
J.J.M. VERBAARSCHOT and P.J. BRUSSAARD \\ Fysisch Laboratorium, Rijksuniversiteit Utrecht, 3508 TA Utrecht, The Netherlands
}

Received 21 November 1978

Revised manuscript received 23 May 1979

The components of shell-model eigenvectors show a non-gaussian distribution that can be derived by the use of the Porter-Thomas proposition.

In nuclear shell-model calculations one is confronted with increasing dimensionalities of the configuration spaces. It makes sense, therefore, to study the statistical properties of the eigenvectors of nuclear states. The distribution of the amplitudes of the eigenvector components of a large-scale shell-model hamiltonian shows a predominance of the small amplitudes.

The distribution is not gaussian, however, but more complicated. In this paper we shall derive an explicit expression for the amplitude distribution from three statistical assumptions. No parameters are to be fitted for the final expression. Several papers (e.g. ref. [1]) have been devoted to studies of the gaussian orthogonal ensemble of matrices. Here, however, we do not consider an ensemble of hamiltonians, but rather study the eigenvalues and eigenvectors of one hamiltonian.

Let the eigenvectors $\psi_{i}$ of the hamiltonian, $H$, be expanded in the basis $\left\{\phi_{\alpha}\right\}(\alpha=1,2, \ldots, N)$ :

$\psi_{i}=\sum_{\alpha=1}^{N} c_{\alpha i} \phi_{\alpha}, \quad i=1, \ldots, N$

The two-body interaction used to construct the hamiltonian for our calculations was the MSDI [2]. The basis states $\phi_{\alpha}$ were taken to span the full sd-shell. For the $J^{\pi}=1 / 2^{+}$states of ${ }^{25} \mathrm{Mg}$ this leads to a dimension 1434 and for the $J^{\pi}=1^{+}$states of ${ }^{22} \mathrm{Na}$ to a dimension 243. For a fixed eigenvector we can plot the values of the coefficients $\left|c_{\alpha i}\right|=\left|\left\langle\phi_{\alpha} \mid \psi_{i}\right\rangle\right|$ versus the values of the diagonal matrix elements $H_{\alpha \alpha}=\left\langle\phi_{\alpha}|H| \phi_{\alpha}\right\rangle$. In fig. 1 such a plot is shown for the lowest $J^{\pi}=1^{+}$ eigenstate of ${ }^{22} \mathrm{Na}$. One observes a secular variation $\left|c_{\alpha i}\right|$ as a function of $H_{\alpha \alpha}$ (solid line) and fluctuations of the actual values of the amplitudes $\left|c_{\alpha i}\right|$ around the average.

These fluctuations will be described by the PorterThomas proposition [3] that the coefficients $c_{\alpha i}$ locally, i.e. for some small neighbourhood of $H_{\alpha \alpha}$, show a normal distribution with zero mean. This gaussian behaviour implies that the variance is proportional to the secular value

$\overline{\left\langle\phi_{\alpha} \mid \psi_{i}\right\rangle^{2}}=(\pi / 2){\overline{\left\langle\phi_{\alpha} \mid \psi_{i}\right\rangle \mid}}^{2}$.

We assume that the diagonal matrix elements

$H_{\alpha \alpha} \equiv\left\langle\phi_{\alpha}|H| \phi_{\alpha}\right\rangle, \quad(\alpha=1,2, \ldots, N)$,

possess a gaussian distribution

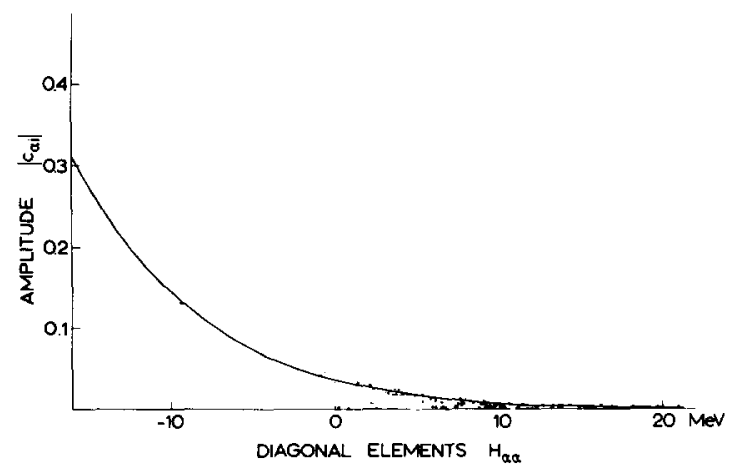

Fig. 1. The amplitudes $\left|\left\langle\phi_{\alpha} \mid \psi_{i}\right\rangle\right|$ versus the diagonal elements $\left\langle\phi_{\alpha}|H| \phi_{\alpha}\right\rangle$. The solid line is the secular variation $\rho_{r}$. 
Table 1

Parameters that determine the gaussian distributions of $H_{\alpha \alpha}$ and $\left|c_{\alpha i}\right|$ (all in MeV). Centroids do not affect the physical results.

\begin{tabular}{|c|c|c|c|c|c|c|c|}
\hline & & ${ }^{22} \mathrm{Na}$ & & & ${ }^{25} \mathrm{Mg}$ & & \\
\hline (a) & $\begin{array}{l}\text { width } \sigma_{0} \\
\text { skewness } \\
\text { excess }\end{array}$ & $\begin{array}{r}7.24 \\
-0.09 \\
-0.30\end{array}$ & & & $\begin{array}{r}7.92 \\
0.06 \\
-0.07\end{array}$ & & \\
\hline (b) & $\begin{array}{l}J^{\pi} \\
\text { width } \sigma_{1} \\
\text { skewness } \\
\text { excess }\end{array}$ & $\begin{array}{r}\left(1^{+}\right)_{1} \\
6.98 \\
0.14 \\
-0.39\end{array}$ & $\begin{array}{r}\left(1^{+}\right)_{2} \\
7.14 \\
0.19 \\
-0.38\end{array}$ & $\begin{array}{r}\left(1^{+}\right)_{3} \\
7.07 \\
0.21 \\
-0.47\end{array}$ & $\begin{array}{c}\left(1 / 2^{+}\right)_{1} \\
8.02 \\
0.12 \\
-0.18\end{array}$ & $\begin{array}{c}\left(1 / 2^{+}\right)_{2} \\
7.51 \\
0.27 \\
-0.12\end{array}$ & $\begin{array}{c}\left(1 / 2^{+}\right)_{3} \\
8.22 \\
0.10 \\
-0.15\end{array}$ \\
\hline
\end{tabular}

$\rho_{0}\left(H_{\alpha \alpha}\right)=\left[N /(2 \pi)^{1 / 2} \sigma_{0}\right] \exp \left[-\left(H_{\alpha \alpha}-E_{0}\right)^{2} / 2 \sigma_{0}^{2}\right]$.

Calculation of skewness and excess tells us that eq. (4) is a good approximation for the actual distribution (see table 1). The secular variation of the values $\left|c_{\alpha i}\right|$ as a function of $H_{\alpha \alpha}$ will be denoted by $\rho_{\mathrm{r}}\left(H_{\alpha \alpha}\right)$. Applying a variant of parametric differentiation [4] one can prove that the product $\rho_{\mathrm{r}}\left(H_{\alpha \alpha}\right) \rho_{0}\left(H_{\alpha \alpha}\right)$ can be approximated by a gaussian

$$
\begin{aligned}
& \rho_{\mathrm{r}}\left(H_{\alpha \alpha}\right) \rho_{0}\left(H_{\alpha \alpha}\right) \equiv \rho_{1}\left(H_{\alpha \alpha}\right) \\
& \quad=\left[M_{0} /(2 \pi)^{1 / 2} \sigma_{1}\right] \exp \left[-\left(H_{\alpha \alpha}-E_{1}\right)^{2} / 2 \sigma_{1}^{2}\right],
\end{aligned}
$$

which possesses the moments

$M_{p}=\sum_{\alpha=1}^{N}\left|c_{\alpha i}\right|\left(H_{\alpha \alpha}\right)^{p}, \quad p=0,1,2$.

Again a calculation of skewness and excess supports the gaussian approximation (see table 1). According to the Porter-Thomas proposition the spread of the values of $\left|c_{\alpha i}\right|$ around the local average $\rho_{\mathrm{r}}\left(H_{\alpha \alpha}\right)$ is described by the normal distribution

$\rho\left(\left|c_{\alpha i}\right|\right)=\left[2 /(2 \pi)^{1 / 2} \sigma\right] \exp \left[-c_{\alpha i}^{2} / 2 \sigma^{2}\right]$,

with the width $\sigma$ determined by the condition

$\frac{2}{(2 \pi)^{1 / 2} \sigma} \int_{0}^{\infty} x \exp \left[-x^{2} / 2 \sigma^{2}\right] \mathrm{d} x=\rho_{\mathrm{r}}\left(H_{\alpha \alpha}\right)$.

This condition yields

$2 \sigma^{2}=\pi \rho_{\mathrm{r}}^{2}\left(H_{\alpha \alpha}\right)$.

The number of amplitudes $\left|c_{\alpha i}\right|$ smaller than some value, say $\Gamma$, in the interval $\left\{H_{\alpha \alpha}, H_{\alpha \alpha}+\Delta H_{\alpha \alpha}\right\}$ is then given by

$\rho_{0}\left(H_{\alpha \alpha}\right) \Delta H_{\alpha \alpha} \int_{0}^{\Gamma} \frac{2}{\pi \rho_{\mathrm{r}}\left(H_{\alpha \alpha}\right)} \exp \left[-x^{2} / \pi \rho_{\mathrm{r}}^{2}\left(H_{\alpha \alpha}\right)\right] \mathrm{d} x$.

With the use of eq. (5) the parameters of the secular variation $\rho_{\mathrm{r}}\left(H_{\alpha \alpha}\right)$ can be expressed in terms of the parameters that determine the distributions $\rho_{0}\left(H_{\alpha \alpha}\right)$ and $\rho_{1}\left(H_{\alpha \alpha}\right)$. One finds

$\rho_{\mathrm{r}}(E)=n_{\mathrm{r}} \exp \left[-\left(E-E_{\mathrm{r}}\right)^{2} / 2 \sigma_{\mathrm{r}}^{2}\right]$,

with

$n_{\mathrm{r}}=\frac{\Sigma_{\alpha}\left|c_{\alpha i}\right| \sigma_{0}}{N \sigma_{1}} \exp \left[\left(E_{1}-E_{0}\right)^{2} / 2\left(\sigma_{0}^{2}-\sigma_{1}^{2}\right)\right]$,

$E_{\mathrm{r}}=\left(\sigma_{0}^{2} E_{1}-\sigma_{1}^{2} E_{0}\right) /\left(\sigma_{0}^{2}-\sigma_{1}^{2}\right), \quad \sigma_{\mathrm{r}}^{-2}=\sigma_{1}^{-2}-\sigma_{0}^{-2}$.

The distribution function of the amplitudes $\left|c_{\alpha i}\right|$ is obtained from expression (10) when the integration over $x=\left|c_{\alpha i}\right|$ is suppressed and an integration over all values of $H_{\alpha \alpha}$ is performed,

$$
\begin{aligned}
& F\left(\left|c_{\alpha i}\right|\right)=\left(2^{1 / 2} / \pi^{3 / 2}\right)\left(N / \sigma_{0} n_{\mathrm{r}}\right) \\
& \quad \times \int_{-\infty}^{\infty} \mathrm{d} E \exp \left\{-\left(E-E_{0}\right)^{2} / 2 \sigma_{0}^{2}+\left(E-E_{\mathrm{r}}\right)^{2} / 2 \sigma_{\mathrm{r}}^{2}\right. \\
& \left.-\left(\left|c_{\alpha i}\right|^{2} / \pi n_{\mathrm{r}}^{2}\right) \exp \left[\left(E-E_{\mathrm{r}}\right)^{2} / \sigma_{\mathrm{r}}^{2}\right]\right\} .
\end{aligned}
$$

In figs. 2 and 3 we show a comparison of results obtained from eq. (12) with the results of an exact counting. This comparison applies to the lowest three $J^{\pi}=1^{+}$states of ${ }^{22} \mathrm{Na}$ and the lowest three $J^{\pi}=1 / 2^{+}$ states of ${ }^{25} \mathrm{Mg}$. It should be emphasized that no parameters are fitted to obtain this reproduction of the exact data. The widths $\sigma_{0}$ and $\sigma_{1}$ are calculated from the diagonal matrix elements $H_{\alpha \alpha}$ and the eigenvector 


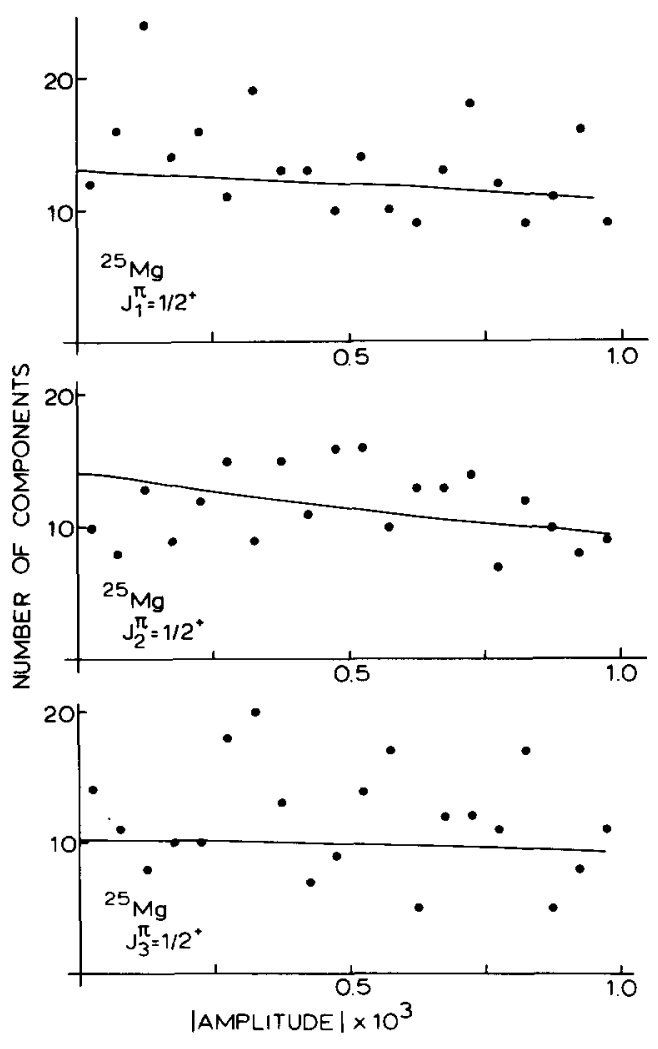

Fig. 2. Distribution of eigenvector components of the lowest three states $J^{\pi}=1 / 2^{+}$of ${ }^{25} \mathrm{Mg}$ for small values of the amplitudes. The solid curve represents the result of eq. (10), multiplied by the size of the interval $5 \times 10^{-4}$.

components $c_{\alpha i}$. In table 1 the numerical values of the widths $\sigma_{0}$ and $\sigma_{1}$ are given, together with the values of skewness and excess. The latter two quantities are small enough to justify the gaussian form of $\rho_{0}$ and $\rho_{1}$. The larger values for ${ }^{22} \mathrm{Na}$ reflect the smaller dimension of the corresponding configuration space. It is seen that the agreement is excellent.

Differentiation of eq. (12) shows that the derivative $F^{\prime}(x)$ at the origin is given by

$F^{\prime}(x=0)=0$, for $\sigma_{\mathrm{r}}^{2}<0$ or $3 \sigma_{0}^{2}<\sigma_{\mathrm{r}}^{2}$,

$F^{\prime}(x=0)=$ undetermined for $0 \leqslant \sigma_{\mathrm{r}}^{2} \leqslant 3 \sigma_{0}^{2}$.

For all calculations that we performed always one of the conditions of eq. (13a) was fulfilled.

Recently Whitehead et al. [5] also have investigated the distribution of eigenvector components. They used
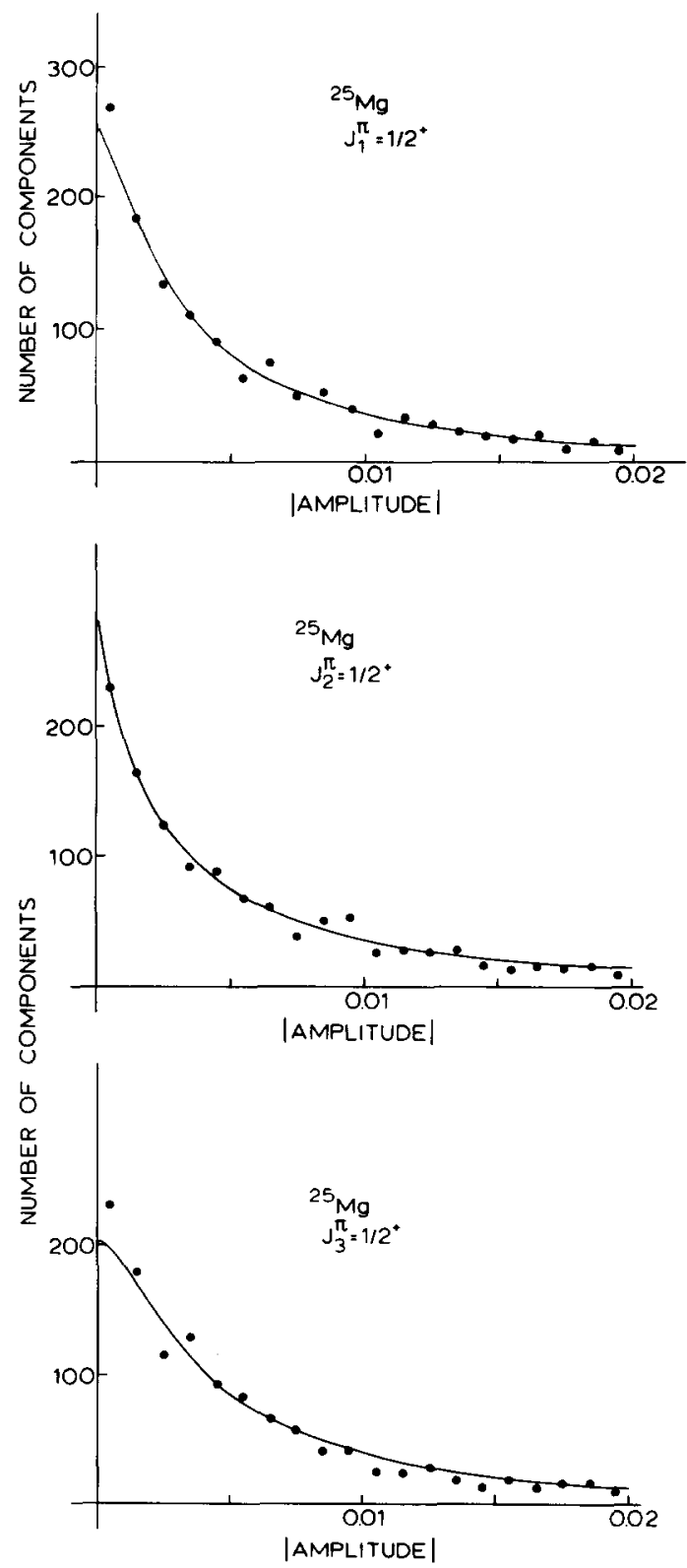

Fig. 3. Distribution of eigenvector components of the lowest three states $J^{\pi}=1 / 2^{+}$of ${ }^{25} \mathrm{Mg}$. The solid curve represents the result of eq. (10), multiplied by the size of the interval $10^{-3}$.

a different approach and considered ensembles of hamiltonians that preserved two-body selection rules. The distribution function they obtained, i.e. the modified Bessel function $K_{0}$, differs from ours, in particular for very small amplitudes $\left|c_{\alpha i}\right|$. It is seen in fig. 4 , how. 

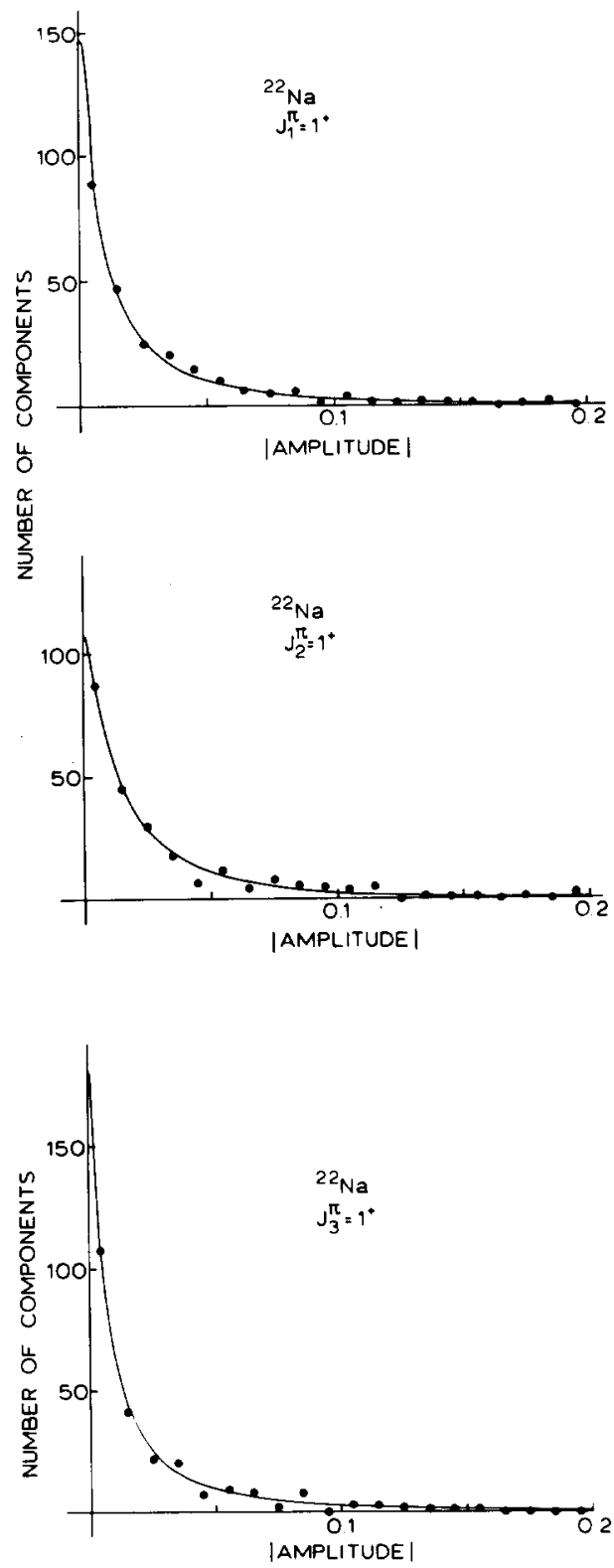

Fig. 4. Distribution of eigenvector components of the lowest three states $J^{\pi}=1^{+}$of ${ }^{22} \mathrm{Na}$. The solid curve represents the result of eq. (10), multiplied by the size of the interval $10^{-2}$. ever, that the distribution function of eigenvector components tends for small amplitudes to a finite value with zero derivative. Such a behaviour is not reproduced by the function $K_{0}$.

It has to be kept in mind that Whitehead et al. obtained their results for the wavefunctions in the $\mathrm{m}$ scheme, whereas our results apply to the amplitudes $\left|c_{\alpha i}\right|$ for the $j j$-coupling scheme. Although the interactions used in the two studies of amplitude distributions are not the same, they both consist of two-body forces and thus we do not expect that their difference will cause a qualitative discrepancy.

The authors would like to thank Drs. A.G.M. van Hees, B.C. Metsch and G.A. Timmer for their assistance with the calculations and useful discussions. This investigation was performed as part of the research programme of the "Stichting voor Fundamenteel Onderzoek der Materie" (FOM) with financial support from the "Nederlandse Organisatie voor Zuiver Wetenschappelijk Onderzoek” (ZWO).

\section{References}

[1] C.E. Porter and N. Rosenzweig, in: Statistical theories of spectra: fluctuations (Academic Press, 1965) p. 235.

[2] P.W.M. Glaudemans, P.J. Brussaard and B.H. Wildenthal, Nucl. Phys. A102 (1967) 593.

[3] C.E. Porter and R.G. Thomas, Phys. Rev. 104 (1956) 483.

[4] F.S. Chang and J.B. French, Phys. Lett. 44B (1973) 131.

[5] R.R. Whitehead, A. Watt, D. Kelvin and A. Conkie, Phys. Lett. 76B (1978) 149. 